\title{
On entropic uncertainty relations in the presence of a minimal length
}

\author{
Alexey E. Rastegin \\ Department of Theoretical Physics, Irkutsk State University, Gagarin Bv. 20, Irkutsk 664003, Russia
}

\begin{abstract}
Entropic uncertainty relations for the position and momentum within the generalized uncertainty principle are examined. Studies of this principle are motivated by the existence of a minimal observable length. Then the position and momentum operators satisfy the modified commutation relation, for which more than one algebraic representation is known. One of them is described by auxiliary momentum so that the momentum and coordinate wave functions are connected by the Fourier transform. However, the probability density functions of the physically true and auxiliary momenta are different. As the corresponding entropies differ, known entropic uncertainty relations are changed. Using differential Shannon entropies, we give a state-dependent formulation with correction term. State-independent uncertainty relations are obtained in terms of the Rényi entropies and the Tsallis entropies with binning. Such relations allow one to take into account a finiteness of measurement resolution.
\end{abstract}

Keywords: generalized uncertainty principle, minimal observable length, Rényi entropy, Tsallis entropy

\section{INTRODUCTION}

One of fundamental problems of modern physics is to describe the gravitation at the quantum level [1]. Today, theoretical efforts are focused on unifying all fundamental interactions into a single theoretical framework. The existence of a minimal observable length has long been suggested due to such studies [2, 3]. It should lead to an effective cutoff in the ultraviolet [4]. String-theoretic arguments also maintain a minimal length effectively in the form of a minimal position uncertainty. There are proposals to investigate observable effects of the minimal length, including astronomical observations [5, 6] and experimental schemes feasible within current technology [7, 8]. The authors of [9 11] discussed measurements in which we may be able to prove effects of quantum gravity. The role of quantum decoherence in modern particle experiments is emphasized in [12].

The uncertainty principle [13] is well known among scientific achievements inspired by the discovery of quanta. Discussion of Heisenberg [13] was rather qualitative in character. There is no general consensus concerning scope and validity of the uncertainty principle [14]. One of topics questioned concerns proper forms of the uncertainty principle beyond the standard quantum mechanics. In many models of quantum gravity and string theory, the Planck length $\ell_{P}=\sqrt{G \hbar / c^{3}} \approx 1.616 \times 10^{-35} \mathrm{~m}$ plays a crucial role. It seems that the very concept of space-time changes its meaning below the Planck scale [15]. Processes around the Planck energy may depend on physical effects of virtual black holes [16]. At this scale, Heisenberg's principle is assumed to be converted into the generalized uncertainty principle (GUP) 17, 18]. Of course, this modification should be consistent with the existence of a minimal observable length. The GUP issue has many aspects that are currently the subject of active researches [19 26].

To get a quantum model with a nonzero minimal uncertainty in position, the commutation relation for position and momentum is modified. Consequences of deformed forms of the commutation relation have attracted much attention in recent years, though their connections with the real world are an open question. In the context of non-relativistic quantum mechanics, the corresponding formalism was developed in [27]. The author of [28] proposed another approach to representation of the position and momentum operators. Being physically equivalent to the representation of [27], it differs in some formal aspects. The above approaches used the momentum representation for dealing with the Schrödinger equation in the GUP case. It was recently shown that the position representation of this case is possible with quasi-nonlinear evolution equation [29]. Path integral quantization corresponding to the deformed algebra was examined in [30]. The authors of [31] thoroughly considered physical assumptions under which the used modifications actually imply a minimal length.

The first explicit derivation of uncertainty relations was given by Kennard [32]: product of the standard deviations of the position and momentum operators cannot be less than the constant equal to $\hbar / 2$. Robertson 33] extended this approach to arbitrary pair of observables. The authors of 34 36] discussed uncertainty relations for photons. Due to lack of well-defined position operator of a photon, their method focuses on the electromagnetic energy distribution in space. Robertson's formulation was later criticized for several reasons [37, 38]. Instead, an entropic formulation of the uncertainty principle has been proposed and motivated [37 40]. Entropic uncertainty relations are currently the subject of active research (see, e.g., the reviews [41 43] and references therein). In finite dimensions, uncertainty relations can be posed in terms of very wide class of entropic functions [4]. Other approaches are based on the sum of variances [45, 46], majorization relations [47 -51] and the technique of effective anti-commutators [52].

Entropic functions provide a clean and flexible tool for characterizing uncertainties in quantum measurements. The first entropic uncertainty relation for the position-momentum pair was derived by Hirschman [53] and later improved 
in [54, 55]. Entropic uncertainty relations in multi-dimensional spaces were given in [56]. In the information-theoretic framework, uncertainty relations in the presence of a quantum memory were formulated [57, 58]. Recently, the generalized uncertainty principle has been analyzed within the entropic approach [59, 60]. These formulations are expressed in terms of the Shannon entropies. On the other hand, more general forms of entropic functions have found use in quantifying uncertainties. Utilities of entropic bounds with a parametric dependence were first emphasized in [38]. In particular, such bounds may allow us to find more exactly the domain of acceptable values for unknown probabilities with respect to known ones.

The aim of the present work is to examine the generalized uncertainty principle in terms of entropic functions of both the Rényi and Tsallis types. We will focus on those questions that were not addressed in this context previously. The paper is organized as follows. In Section II] we review preliminary material including details of the used representation of the position and momentum operators. A state-dependent formulation of the generalized uncertainty principle is obtained in Section III with the use of Shannon entropies. In Section IV we obtain state-independent uncertainty relations in terms of the Rényi entropies and, with appropriate binning, the Tsallis entropies. In Section $\mathrm{V}$. we conclude the paper with a brief summary of results.

\section{PRELIMINARIES}

In this section, we recall the required material and describe the notation. For convenience, we will use the wavenumber operator $\hat{k}$ instead of the momentum $\hat{p}=\hbar \hat{k}$. Another viewpoint is that the used system of units provides $\hbar=1$. As we will focus on the momentum representation, the following fact should be mentioned. In particular, the considered approach deals with a linear equation which contains momentum derivatives of every order. On the other hand, the nonlinear equation of [29] involves only second order spatial derivatives. The method of [29] also gives a new perspective of links between spacetime symmetries and quantum linearity [61].

In the one-dimensional case, the position and momentum operators obey the deformed commutation relation [27]. We rewrite the commutation relation as

$$
[\hat{x}, \hat{k}]=\mathrm{i}\left(\mathbb{1}+\beta \hat{k}^{2}\right) \text {. }
$$

Here, the positive parameter $\beta$ is assumed to be rescaled by factor $\hbar^{2}$ from its usual sense, and $\mathbb{1}$ is the identity operator. In the limit $\beta \rightarrow 0$, the formula (1) gives the well-known commutation relation of ordinary quantum mechanics. Due to the Robertson formulation [33], the standard deviations in the prepared state $\hat{\rho}$ obey

$$
\Delta \hat{A} \Delta \hat{B} \geq\left|\frac{1}{2}\langle[\hat{A}, \hat{B}]\rangle_{\hat{\rho}}\right| .
$$

By $\langle\hat{A}\rangle_{\hat{\rho}}=\operatorname{Tr}(\hat{A} \hat{\rho})$, we mean the quantum-mechanical expectation value. The authors of [62] recently examined statedependent uncertainty relations that are tighter than the Roberson-Schrödinger uncertainty relation. Combining (1) with (2) then leads to the inequality

$$
\Delta \hat{x} \Delta \hat{k} \geq \frac{1}{2}\left(1+\beta\left\langle\hat{k}^{2}\right\rangle_{\hat{\rho}}\right) \geq \frac{1}{2}\left(1+\beta(\Delta \hat{k})^{2}\right) .
$$

The principal parameter $\beta$ is positive and independent of $\Delta \hat{x}$ and $\Delta \hat{k}$ [27]. It follows from (3) that $\Delta \hat{x}$ does not exceed the square root of $\beta$.

Following [28], we will use the auxiliary wavenumber operator $\hat{q}$. Let $\hat{x}$ and $\hat{q}$ be self-adjoint operators that obey $[\hat{x}, \hat{q}]=i \mathbb{1}$. In the $q$-space, the action of $\hat{q}$ results in multiplying a wave function $\varphi(q)$ by $q$, whereas $\hat{x} \varphi(q)=\mathrm{i} \mathrm{d} \varphi / \mathrm{d} q$. The author of [28] proposed the representation

$$
\hat{k}=\frac{1}{\sqrt{\beta}} \tan (\sqrt{\beta} \hat{q}) .
$$

The auxiliary wavenumber satisfies the ordinary commutation relation but ranges between $\pm q_{0}(\beta)= \pm \pi /(2 \sqrt{\beta})$. The function $q \mapsto k=\tan (\sqrt{\beta} q) / \sqrt{\beta}$ gives a one-to-one correspondence between $q \in\left(-q_{0} ;+q_{0}\right)$ and $k \in(-\infty ;+\infty)$. So, the eigenvalues of $\hat{k}$ fully cover the real axis. The above representation is formally self-adjoint. However, one provides only $\mathcal{D}(\hat{x}) \subset \mathcal{D}\left(\hat{x}^{\dagger}\right)$, though $\mathcal{D}(\hat{k})=\mathcal{D}\left(\hat{k}^{\dagger}\right)$ due to von Neumann's theorem [28].

For a pure state, we actually have three wave functions $\phi(k), \varphi(q)$, and $\psi(x)$. The auxiliary wave function $\varphi(q)$ provides a convenient mathematical tool as connected with $\psi(x)$ via the Fourier transform. Let the eigenkets $|q\rangle$ of $\hat{q}$ be normalized through Dirac's delta function and obey the completeness relation

$$
\int_{-q_{0}}^{+q_{0}} \mathrm{~d} q|q\rangle\langle q|=\mathbb{1}
$$


In the $q$-space, the eigenfunctions of $\hat{x}$ appear as $\langle q \mid x\rangle=\exp (-i q x) / \sqrt{2 \pi}$. Combining this with (5), any wave function in the coordinate space is expressed as

$$
\psi(x)=\frac{1}{\sqrt{2 \pi}} \int_{-q_{0}}^{+q_{0}} \exp (+\mathbf{i} q x) \varphi(q) \mathrm{d} q .
$$

Wave functions in the $q$ - and $x$-spaces are connected by the Fourier transform [28],

$$
\varphi(q)=\frac{1}{\sqrt{2 \pi}} \int_{-\infty}^{+\infty} \exp (-\mathbf{i} q x) \psi(x) \mathrm{d} x .
$$

The only distinction from ordinary quantum mechanics is that each wave function $\varphi(q)$ in the $q$-space should be formally treated as 0 for all $|q|>q_{0}(\beta)$.

Using the above connection, the author of [59] claimed the following. The uncertainty relation of Beckner [54] and of Białynicki-Birula and Mycielski [55] is still valid for the generalized uncertainty principle. However, wave functions in the $q$-space play only auxiliary role. In the GUP case, the physically legitimate wavenumber and momentum involved in the relation (11) are described by wavefunctions in the $k$-space. An actual distribution of physical wavenumber values is therefore determined with respect to $\phi(k)$ instead of $\varphi(q)$. Let us consider the probability that momentum lies between two prescribed values. Due to the one-to-one correspondence between $k$ and $q$, there is a bijection between the intervals $\left(k_{1} ; k_{2}\right)$ and $\left(q_{1} ; q_{2}\right)$. Thus, the probability can be expressed as

$$
\int_{k_{1}}^{k_{2}}|\phi(k)|^{2} \mathrm{~d} k=\int_{q_{1}}^{q_{2}}|\varphi(q)|^{2} \mathrm{~d} q
$$

whence $|\phi(k)|^{2} \mathrm{~d} k=|\varphi(q)|^{2} \mathrm{~d} q$. More generally, two probability density functions $u(k)$ and $v(q)$ are connected as $u(k) \mathrm{d} k=v(q) \mathrm{d} q$, in another form

$$
u(k)=\frac{v(q)}{1+\beta k^{2}} .
$$

For pure states, when $u(k)=|\phi(k)|^{2}$ and $v(q)=|\varphi(q)|^{2}$, the formula (9) is obvious. It is directly extended to mixed states by the spectral decomposition.

In reality, we do not deal with the probability density functions $u(k)$ and $w(x)$ immediately. Eigenkets of unbounded operators, say $|k\rangle$ and $|x\rangle$, are not elements of the Hilbert space [63]. Instead, we may deal with narrow distributions that are of a finite but small width. Here, a finiteness of detector resolution should be addressed [64, 65]. Measuring or preparing a state with the particular value $\xi$ of position, one is affected by some vicinity of $\xi$. In this way, we refer to generalized quantum measurements [66]. Unsharpness of such measurements in the context of entropic uncertainty relations was studied in [67]. Dealing with a finite-resolution measurement of the legitimate wavenumber, the set $\mathcal{K}=\{|k\rangle\langle k|\}$ is replaced with some set $\mathcal{M}$ of operators of the form

$$
\hat{M}(\zeta):=\int_{-\infty}^{+\infty} \mathrm{d} k f(\zeta-k)|k\rangle\langle k|
$$

An acceptance function $\zeta \mapsto f(\zeta)$ obeys the normalization condition, so that $\int_{-\infty}^{+\infty}|f(\zeta)|^{2} \mathrm{~d} \zeta=1$. Then operators of the form (10) lead to a non-projective resolution of the identity, namely

$$
\int_{-\infty}^{+\infty} \mathrm{d} \zeta \hat{M}(\zeta)^{\dagger} \hat{M}(\zeta)=\mathbb{1}
$$

For the input $\hat{\rho}$, the measurement results in the probability density function

$$
U(\zeta)=\operatorname{Tr}\left(\hat{M}(\zeta)^{\dagger} \hat{M}(\zeta) \hat{\rho}\right)=\int_{-\infty}^{+\infty}|f(\zeta-k)|^{2} u(k) \mathrm{d} k,
$$

dealt with instead of $u(k)$. If the acceptance function is sufficiently narrow, we will obtain a good "footprint" of $u(k)$. Let $\xi \mapsto g(\xi)$ be another acceptance function that also obeys the normalization condition. A finite-resolution measurement of the position is described by the set $\mathcal{N}$ of operators

$$
\hat{N}(\xi):=\int_{-\infty}^{+\infty} \mathrm{d} x g(\xi-x)|x\rangle\langle x| .
$$


Here, the projective resolution $\mathcal{X}=\{|x\rangle\langle x|\}$ is replaced with $\mathcal{N}=\{\hat{N}(\xi)\}$. Instead of $w(x)$, we actually deal with the probability density function

$$
W(\xi)=\operatorname{Tr}\left(\hat{N}(\xi)^{\dagger} \hat{N}(\xi) \hat{\rho}\right)=\int_{-\infty}^{+\infty}|g(\xi-x)|^{2} w(x) \mathrm{d} x .
$$

For good acceptance functions, a distortion of statistics will be small. The Gaussian distribution is a typical form of such functions [64]. It is natural to assume that a behavior of acceptance functions is qualitatively similar.

\section{A STATE-DEPENDENT BOUND ON THE SUM OF SHANNON ENTROPIES}

In this section, we will pose the generalized uncertainty principle into a lower bound on the sum of Shannon entropies. The usual lower bound is shown to be added by a state-dependent correction term. We begin with differential entropies of the Shannon type. For the given pre-measurement state $\hat{\rho}$, the wavenumber is distributed according to the probability density function $u(k)=\langle k|\hat{\rho}| k\rangle$, where $k \in(-\infty ;+\infty)$ and the eigenkets $|k\rangle$ are normalized through Dirac's delta function. These kets form a projective resolution $\mathcal{K}=\{|k\rangle\langle k|\}$ of the identity. Then the differential entropy is defined as

$$
H_{1}(\mathcal{K} \mid \hat{\rho}):=-\int_{-\infty}^{+\infty} u(k) \ln u(k) \mathrm{d} k
$$

Similarly, we determine entropies for other continuous variables of interest. According to (5), the eigenkets $|q\rangle$ form another resolution, $\mathcal{Q}=\{|q\rangle\langle q|\}$, whence we write $v(q)=\langle q|\hat{\rho}| q\rangle$ and

$$
H_{1}(\mathcal{Q} \mid \hat{\rho}):=-\int_{-q_{0}}^{+q_{0}} v(q) \ln v(q) \mathrm{d} q .
$$

The measurement of position is specified by the resolution $\mathcal{X}=\{|x\rangle\langle x|\}$. With the probability density function $w(x)=\langle x|\hat{\rho}| x\rangle$, one gets the entropy $H_{1}(\mathcal{X} \mid \hat{\rho})$.

Using (9), we get the link between (15) and (16), namely

$$
\begin{aligned}
H_{1}(\mathcal{K} \mid \hat{\rho}) & =-\int_{-q_{0}}^{+q_{0}} v(q) \ln v(q) \mathrm{d} q+\int_{-\infty}^{+\infty} u(k) \ln \left(1+\beta k^{2}\right) \mathrm{d} k \\
& =H_{1}(\mathcal{Q} \mid \hat{\rho})+\left\langle\ln \left(\mathbb{1}+\beta \hat{k}^{2}\right)\right\rangle_{\hat{\rho}}
\end{aligned}
$$

Concerning entropic uncertainty relations in the GUP case, the following fact was noticed [59]. Due to (60) and (77), wave functions in the auxiliary $q$-space are connected with coordinate wave functions via the Fourier transform. In this regard, one merely restricts a consideration to those functions $\varphi(q)$ that are zero beyond the range $q \in\left(-q_{0} ;+q_{0}\right)$. Hence, we can apply the entropic uncertainty relation of Beckner [54] and of Białynicki-Birula and Mycielski [55],

$$
H_{1}(\mathcal{Q} \mid \hat{\rho})+H_{1}(\mathcal{X} \mid \hat{\rho}) \geq \ln (e \pi) .
$$

Although the bound (18) was first formulated for pure states, its extension to impure ones is not difficult. The author of [59] also calculated entropies of (18) for stationary states of the harmonic oscillator in the presence of a minimal length. In arbitrary dimensions, the energy eigenvalues and eigenfunctions of the harmonic oscillator with the modified commutation relation were found in [68].

We already mentioned that the legitimate momentum of the commutation relation (11) appears as $\hbar \hat{k}$. The wavenumber operator $\hat{q}$ is only a useful auxiliary tool. Instead of $H_{1}(\mathcal{Q} \mid \hat{\rho})$, the Shannon entropy $H_{1}(\mathcal{K} \mid \hat{\rho})$ should be used to quantify a momentum uncertainty in the presence of a minimal observable length. Combining (17) with (18) gives the basic result of this section,

$$
H_{1}(\mathcal{K} \mid \hat{\rho})+H_{1}(\mathcal{X} \mid \hat{\rho}) \geq \ln (e \pi)+\left\langle\ln \left(\mathbb{1}+\beta \hat{k}^{2}\right)\right\rangle_{\hat{\rho}} .
$$

The second quantity in the right-hand side of (19) is a correction of lower entropic bound in the GUP case. It is similar to the correction term appearing in Robertson's formulation (3).

In practice, we deal with the probability densities (12) and (14) after masking by acceptance functions. Substituting each of these densities into the right-hand side of (15), we obtain the differential entropies $H_{1}(\mathcal{M} \mid \hat{\rho})$ and $H_{1}(\mathcal{N} \mid \hat{\rho})$. The entropic bound with the correction term remains valid for these entropies, i.e.,

$$
H_{1}(\mathcal{M} \mid \hat{\rho})+H_{1}(\mathcal{N} \mid \hat{\rho}) \geq \ln (e \pi)+\left\langle\ln \left(\mathbb{1}+\beta \hat{k}^{2}\right)\right\rangle_{\hat{\rho}} .
$$


We can prove (20) by means of one result for integral mean values with a weight function (see theorem 204 of the book [69]). Let the weight function $\lambda(x)$ be normalized. If $\Phi^{\prime \prime}(t)$ is positive for all $t$ between inf $w(x)$ and $\sup w(x)$, then

$$
\Phi\left(\int \lambda(x) w(x) \mathrm{d} x\right) \leq \int \lambda(x) \Phi(w(x)) \mathrm{d} x .
$$

This result needs a lot of technical conditions, which are all fulfilled in our case. If $\Phi^{\prime \prime}(t)$ is negative, then the inequality (21) should be rewritten in opposite direction. Combining concavity of the function $t \mapsto-t \ln t$ with properties of acceptance functions finally leads to the relations [65]

$$
H_{1}(\mathcal{M} \mid \hat{\rho}) \geq H_{1}(\mathcal{K} \mid \hat{\rho}), \quad H_{1}(\mathcal{N} \mid \hat{\rho}) \geq H_{1}(\mathcal{X} \mid \hat{\rho})
$$

It is physically natural that an additional masking of acceptance functions cannot reduce the amount of uncertainty. Combining (19) with (22) at once gives the claim (20).

The results (19) and (20) lead to entropic uncertainty relations with binning. Using some discretization, we will always have positive entropic functions and take into account typical experimental settings. In the case of position measurements, values $x_{j}$ mark the ends of intervals $\delta x_{j}=x_{j+1}-x_{j}$. We now deal with probabilities

$$
p_{j}^{(\delta)}:=\int_{x_{j}}^{x_{j+1}} w(x) \mathrm{d} x
$$

which give the discrete distribution $p_{\mathcal{X}}^{(\delta)}$ with the Shannon entropy $H_{1}\left(p_{\mathcal{X}}^{(\delta)} \mid \hat{\rho}\right)$. For each $j$, we apply the above theorem for integral means, where integrals are taken over the range between $x_{j}$ and $x_{j+1}$ and $\lambda=1 / \delta x_{j}$. As the function $t \mapsto-t \ln t$ is concave, the inequality (21) should be rewritten in opposite direction. By doing some algebra, we have

$$
-p_{j}^{(\delta)} \ln p_{j}^{(\delta)} \geq-\int_{x_{j}}^{x_{j+1}} w(x) \ln w(x) \mathrm{d} x-p_{j}^{(\delta)} \ln \delta x_{j} .
$$

Using $-\ln \delta x_{j} \geq-\ln \delta x$ with $\delta x=\max \delta x_{j}$, we sum (24) with respect to $j$ and get

$$
H_{1}\left(p_{\mathcal{X}}^{(\delta)} \mid \hat{\rho}\right) \geq H_{1}(\mathcal{X} \mid \hat{\rho})-\ln \delta x
$$

By a parallel argument, $H_{1}\left(p_{\mathcal{K}}^{(\delta)} \mid \hat{\rho}\right) \geq H_{1}(\mathcal{K} \mid \hat{\rho})-\ln \delta k$, where $\delta k$ is the maximal size of wavenumber bins. Combining these inequalities with (19) gives

$$
H_{1}\left(p_{\mathcal{K}}^{(\delta)} \mid \hat{\rho}\right)+H_{1}\left(p_{\mathcal{X}}^{(\delta)} \mid \hat{\rho}\right) \geq \ln \left(\frac{e \pi}{\delta k \delta x}\right)+\left\langle\ln \left(\mathbb{1}+\beta \hat{k}^{2}\right)\right\rangle_{\hat{\rho}} .
$$

Converting (20) into uncertainty relations with binning is obvious. We refrain from presenting the details here. The first summand in the right-hand side of (26) appears just as in entropic uncertainty relations proved in [70]. The second summand reflects the presence of a minimal length.

From the physical viewpoint, the parameter $\beta$ is assumed to be small. Taking the linear order in $\beta$, we have

$$
\left\langle\ln \left(\mathbb{1}+\beta \hat{k}^{2}\right)\right\rangle_{\hat{\rho}}=\beta\left\langle\hat{k}^{2}\right\rangle_{\hat{\rho}}+O\left(\beta^{2}\right)
$$

Thus, the correction term to the entropic bound is the doubled correction term of Robertson's formulation (3). These correction terms inspired by the GUP are physically significant together with each other. They are always nonzero for wave packets of a finite width. Further, the correction to the entropic bound is bounded from above by the logarithm of the doubled Robertson bound. For any quantum state, we have

$$
\left\langle\ln \left(\mathbb{1}+\beta \hat{k}^{2}\right)\right\rangle_{\hat{\rho}} \leq \ln \left(1+\beta\left\langle\hat{k}^{2}\right\rangle_{\hat{\rho}}\right) .
$$

It can be proved on the base of the theorem (21) applied properly. Of course, any upper bound on the correction term is not so interesting. Nevertheless, the result (28) characterizes a scale in which the correction term may vary. Moreover, the two sides of (28) coincide in the term $\sim \beta$ too. 


\section{RÉNYI FORMULATION OF ENTROPIC UNCERTAINTY RELATIONS}

In this section, we formulate state-independent uncertainty bounds in terms of Rényi entropies and, with binning, in terms of Tsallis entropies. We aim to take into account the presence of a minimal length together with an alteration of statistics due to a finite resolution of the measurements. When acceptance functions of measurement apparatuses are sufficiently spread, the existing entropic lower bounds can be improved.

For strictly positive $\alpha \neq 1$, the Rényi generalization of $H_{1}(\mathcal{X} \mid \hat{\rho})$ is written as

$$
R_{\alpha}(\mathcal{X} \mid \hat{\rho}):=\frac{1}{1-\alpha} \ln \left(\int_{-\infty}^{+\infty} w(x)^{\alpha} \mathrm{d} x\right) .
$$

The standard differential entropy $H_{1}(\mathcal{X} \mid \hat{\rho})$ is obtained in the limit $\alpha \rightarrow 1$. It will be convenient to use the quantity

$$
\|w\|_{\alpha}=\left(\int_{-\infty}^{+\infty} w(x)^{\alpha} \mathrm{d} x\right)^{1 / \alpha},
$$

where $\alpha>0$ and $w(x)$ is positive valued. It is similar to the definition of some norms, but gives a legitimate one only for $\alpha \geq 1$. The Rényi entropies of discrete probability distributions are more conventional [71]. For the distribution with probabilities (23), its Rényi $\alpha$-entropy is defined as

$$
R_{\alpha}\left(p_{\mathcal{X}}^{(\delta)} \mid \hat{\rho}\right):=\frac{\alpha}{1-\alpha} \ln \left\|p_{\mathcal{X}}^{(\delta)}\right\|_{\alpha}
$$

where the discrete counterpart of (30) reads

$$
\left\|p_{\mathcal{X}}^{(\delta)}\right\|_{\alpha}=\left(\sum_{j}\left[p_{j}^{(\delta)}\right]^{\alpha}\right)^{1 / \alpha}
$$

Entropic uncertainty relations with binning will be derived by means of entropies of the form (31). The Tsallis entropies [72] form another especially important family of generalized entropies. In the discrete case, one defines

$$
H_{\alpha}\left(p_{\mathcal{X}}^{(\delta)} \mid \hat{\rho}\right):=\frac{1}{1-\alpha}\left(\left\|p_{\mathcal{X}}^{(\delta)}\right\|_{\alpha}^{\alpha}-1\right)
$$

where $0<\alpha \neq 1$. In a similar way, the differential Tsallis entropy is defined via of quantities of the form (30). For certain reasons, we will obtain Tsallis-entropy uncertainty relations only with binning. Basic properties of generalized entropies with application to quantum physics are considered in [73].

As the functions $\varphi(q)$ and $\psi(x)$ are connected by (6) and (7), certain norms of them obey Beckner's inequalities [54]. Let positive parameters $\alpha$ and $\gamma$ satisfy the condition $1 / \alpha+1 / \gamma=2$. For $\alpha>1>\gamma$, we have the inequality

$$
\|v\|_{\alpha} \leq\left(\frac{1}{\varkappa \pi}\right)^{(1-\gamma) / \gamma}\|w\|_{\gamma},
$$

and its "twin" with swapped $v$ and $w$ [74]. Here, the square of $\varkappa$ is expressed as

$$
\varkappa^{2}=\alpha^{1 /(\alpha-1)} \gamma^{1 /(\gamma-1)} .
$$

For a pure state, the above probability density functions read $v(q)=|\varphi(q)|^{2}$ and $w(x)=|\psi(x)|^{2}$. Inequalities of the form (34) are first derived for pure states and then easily extended to impure ones [74, 75].

Using (34) and its twin, we could restate known uncertainty relations in terms of generalized entropies. These relations remain unchanged, only if norms in the momentum space are calculated with the density $v(q)$. However, the probability density function $u(k)$ is primary in the GUP case. As was discussed above, this distribution is additionally masked with some acceptance function. Turning (34) into inequalities for actually registered densities $U(\zeta)$ and $W(\xi)$, we will take into account both aspects of the problem. Combining (12) with $u(k) \mathrm{d} k=v(q) \mathrm{d} q$ leads to

$$
U(\zeta)=\int_{-q_{0}}^{+q_{0}} \mu(\zeta, q) v(q) \mathrm{d} q
$$

where $\mu(\zeta, q)=|f(\zeta-k(q))|^{2}$. The latter is sub-normalized with respect to $q$ in the sense of

$$
J(\zeta)=\int_{-q_{0}}^{+q_{0}} \mu(\zeta, q) \mathrm{d} q=\int_{-\infty}^{+\infty} \frac{|f(\zeta-k)|^{2}}{1+\beta k^{2}} \mathrm{~d} k \leq \int_{-\infty}^{+\infty}|f(\zeta-k)|^{2} \mathrm{~d} k
$$


i.e., $J(\zeta) \leq 1$. To relate $\|U\|_{\alpha}$ and $\|v\|_{\alpha}$, we use (21) with the normalized weight $\lambda(q)=J(\zeta)^{-1} \mu(\zeta, q)$. For $\alpha>1$, the function $t \mapsto t^{\alpha}$ has positive second derivative. Then the theorem (21) leads to

$$
J(\zeta)^{1-\alpha} U(\zeta)^{\alpha} \leq \int_{-q_{0}}^{+q_{0}}|f(\zeta-k(q))|^{2} v(q)^{\alpha} \mathrm{d} q .
$$

Let us introduce the quantity

$$
S_{f}:=\sup _{\zeta} J(\zeta)=\sup _{\zeta} \int_{-\infty}^{+\infty} \frac{|f(\zeta-k)|^{2}}{1+\beta k^{2}} \mathrm{~d} k .
$$

When $\alpha>1$, we have $S_{f}^{1-\alpha} \leq J(\zeta)^{1-\alpha}$ for all $\zeta$. Substituting the latter into (38) and integrating over $\zeta$, we finally get

$$
S_{f}^{(1-\alpha) / \alpha}\|U\|_{\alpha} \leq\|v\|_{\alpha}
$$

The probability density functions $W(\xi)$ and $w(x)$ are connected by (14). For $0<\gamma<1$, the function $t \mapsto t^{\gamma}$ has negative second derivative. By a parallel argument, we obtain $\|w\|_{\gamma} \leq\|W\|_{\gamma}$. It then follows from (34) that

$$
\|U\|_{\alpha} \leq\left(\frac{S_{f}}{\varkappa \pi}\right)^{(1-\gamma) / \gamma}\|W\|_{\gamma}
$$

where $1 / \alpha+1 / \gamma=2$ and $\alpha>1>\gamma$. At the last step, we take into account that $(1-\alpha) / \alpha=(\gamma-1) / \gamma$. By a parallel argument, the inequality between $\|w\|_{\alpha}$ and $\|v\|_{\gamma}$ is transformed into

$$
\|W\|_{\alpha} \leq\left(\frac{S_{f}}{\varkappa \pi}\right)^{(1-\gamma) / \gamma}\|U\|_{\gamma}
$$

under the same conditions on $\alpha$ and $\gamma$. The formulas (41) and (42) immediately lead to uncertainty relations in terms of Rényi entropies. To do so, we take the logarithm of (41) and (42) and then use the expression of entropies via $\|U\|_{\alpha}$ and so on. In the presence of a minimal observable length, we obtain

$$
R_{\alpha}(\mathcal{M} \mid \hat{\rho})+R_{\gamma}(\mathcal{N} \mid \hat{\rho}) \geq \ln \left(\frac{\varkappa \pi}{S_{f}}\right)
$$

where $1 / \alpha+1 / \gamma=2$ and $S_{f}$ is defined by (39). The presented entropic bound is independent of the measured state. For the fixed entropic orders $\alpha$ and $\gamma$, the right-hand side of (43) depends only on $S_{f}$, i.e., on the momentum acceptance function and the minimal-length parameter $\beta$. As follows from (35), the term $\varkappa$ increases from $\varkappa=2$ for $\gamma=1 / 2$ up to $\varkappa=e$ for $\gamma=1$. For the particular choice $\alpha=\gamma=1$, we therefore have

$$
H_{1}(\mathcal{M} \mid \hat{\rho})+H_{1}(\mathcal{N} \mid \hat{\rho}) \geq \ln \left(\frac{e \pi}{S_{f}}\right)
$$

Let us take the probability distributions $p_{\mathcal{M}}^{(\delta)}$ and $p_{\mathcal{N}}^{(\delta)}$ obtained by discretization of the densities $U(\zeta)$ and $W(\xi)$ with respect to chosen marks on the $\zeta$ - and $\xi$-axes. Probabilities are determined by integrals similarly to (23). Assuming $1 / \alpha+1 / \gamma=2$ and $\alpha>1>\gamma$, one can convert (41) and (42) into the inequality

$$
\left\|p_{\mathcal{M}}^{(\delta)}\right\|_{\alpha} \leq\left(\frac{S_{f} \delta \zeta \delta \xi}{\varkappa \pi}\right)^{(1-\gamma) / \gamma}\left\|p_{\mathcal{N}}^{(\delta)}\right\|_{\gamma}
$$

and its "twin" with swapped $p_{\mathcal{M}}^{(\delta)}$ and $p_{\mathcal{N}}^{(\delta)}$. For $1 / \alpha+1 / \gamma=2$, we finally obtain entropic uncertainty relations with binning,

$$
R_{\alpha}\left(p_{\mathcal{M}}^{(\delta)} \mid \hat{\rho}\right)+R_{\gamma}\left(p_{\mathcal{N}}^{(\delta)} \mid \hat{\rho}\right) \geq \ln \left(\frac{\varkappa \pi}{S_{f} \delta \zeta \delta \xi}\right)
$$

To derive uncertainty relations in terms of Rényi entropies, purely algebraic operations were used. The case of Tsallis entropies is not so immediate. We will adopt the method of [76], where the minimization problem was examined. It is essential for optimization that norm-like functionals of discrete probability distributions obey inequalities of the form

$$
\left\|p_{\mathcal{M}}^{(\delta)}\right\|_{\alpha} \leq 1 \leq\left\|p_{\mathcal{M}}^{(\delta)}\right\|_{\gamma}
$$


where $\alpha>1>\gamma$. For probability density functions, such inequalities do not hold always. Hence, we will consider Tsallis entropies only with binning. For $0<\alpha \neq 1$ and $y>0$, we put the $\alpha$-logarithm $\ln _{\alpha}(y):=\left(y^{1-\alpha}-1\right) /(1-\alpha)$. The Tsallis entropies of the corresponding discrete distributions satisfy

$$
H_{\alpha}\left(p_{\mathcal{M}}^{(\delta)} \mid \hat{\rho}\right)+H_{\gamma}\left(p_{\mathcal{N}}^{(\delta)} \mid \hat{\rho}\right) \geq \ln _{\nu}\left(\frac{\varkappa \pi}{S_{f} \delta \zeta \delta \xi}\right)
$$

where $1 / \alpha+1 / \gamma=2$ and $\nu=\max \{\alpha, \gamma\}$. It follows from (37) that $S_{f} \leq 1$. Hence, the uncertainty relations (43), (46), and (48) remain valid with 1 instead of $S_{f}$.

The presented reasons also give an evidence that the existence of a minimal length will increase state-independent entropic bounds. This increasing takes place, when the quantity (39) is strictly less than 1. As an example, we consider the momentum acceptance function

$$
|f(\zeta)|^{2}=\frac{1}{\sigma \sqrt{2 \pi}} \exp \left(-\frac{\zeta^{2}}{2 \sigma^{2}}\right) .
$$

Substituting (49) into (37) gives

$$
S_{f} \leq \frac{1}{\sigma \sqrt{2 \pi}} \int_{-\infty}^{+\infty} \frac{\mathrm{d} k}{1+\beta k^{2}}=\sqrt{\frac{\pi}{2 \sigma^{2} \beta}}
$$

The above entropic relations can all be recast with the right-hand side of (50) instead of $S_{f}$. It is strictly less than 1 , whenever one has $\sigma>\sqrt{\pi /(2 \beta)}$. If the momentum acceptance function is not sufficiently narrow, we will deal with increasing of state-independent lower bounds in entropic uncertainty relations. Addition amount of uncertainty may be due to limitations of measurement resolution. It seems that more accurate bounds on (39) could be obtained. This question could be a subject of separate investigation.

\section{CONCLUSIONS}

We have examined the question how entropic uncertainty relations for position and momentum are changed in the GUP case. Our approach is based on the formally self-adjoint representation proposed in [28]. To describe the momentum, two different operators are used here. We showed that lower entropic bounds are increased, when the physically true momentum is dealt with. Just this momentum obeys the modified commutation relation. Increasing of entropic bounds has been formulated in two distinct ways. The known lower bound on the sum of differential Shannon entropies is added by the expectation value of certain operator. Taking the linear order in $\beta$, this correction term coincides with the doubled correction inspired in Robertson's formulation. We further formulated state-independent uncertainty relations in terms of generalized entropies, both differential and with binning. A finiteness of measurement resolution was naturally involved into the consideration. It has been shown that entropic lower bounds for actually measured distributions are generally increased in the GUP case.

[1] C. Rovelli, Quantum Gravity. Cambridge University Press, Cambridge (2004).

[2] L.J. Garay, Int. J. Mod. Phys. A 10 (1995) 145.

[3] S. Hossenfelder, Living Rev. Relativity 16 (2013) 2.

[4] A. Kempf, G. Mangano, Phys. Rev. D 55 (1997) 7909.

[5] G. Amelino-Camelia, J. Ellis, N.E. Mavromatos, D.V. Nanopoulos, S. Sarkar, Nature 393 (1998) 763.

[6] U. Jacob, T. Piran, Nature Phys. 3 (2007) 87.

[7] I. Pikovski, M.R. Vanner, M. Aspelmeyer, M. Kim, Č. Brukner, Nature Phys. 8 (2012) 393.

[8] F. Marin, F. Marino, M. Bonaldi, M. Cerdonio, L. Conti, P. Falferi, R. Mezzena, A. Ortolan, G.A. Prodi, L. Taffarello, G. Vedovato, A. Vinante, J.-P. Zendri, Nature Phys. 9 (2013) 71.

[9] A.F. Ali, M.M. Khalil, E.C. Vagenas, arXiv:1510.06365 [quant-ph].

[10] K. Bousso, I. Halpern, J. Koeller, Phys. Rev. D 94 (2016) 064047.

[11] R. Howl, L. Hackermüller, D.E. Bruschi, I. Fuentes, arXiv:1607.06666 [quant-ph].

[12] A.K. Alok, S. Banerjee, S.U. Sankar, Phys. Lett. B 749 (2015) 94.

[13] W. Heisenberg, Z. Phys. 43 (1927) 172.

[14] P. Busch, T. Heinonen, P.J. Lahti, Phys. Rep. 452 (2007) 155.

[15] D. Amati, M. Ciafaloni, G. Veneziano, Phys. Lett. B 216 (1989) 41. 
[16] M. Faizal, JETP 114 (2012) 400.

[17] F. Scardigli, Phys. Lett. B 452 (1999) 39.

[18] C. Bambi, Class. Quantum Grav. 25 (2008) 105003.

[19] A. Tawfik, JCAP 07(2013) (2013) 040.

[20] A. Tawfik, A. Diab, Int. J. Mod. Phys. A 23 (2014) 1430025.

[21] M. Faizal, B. Majumder, Ann. Phys. 357 (2015) 49.

[22] M. Faizal, M.M. Khalil, Int. J. Mod. Phys. A 30 (2015) 1550144.

[23] S. Gangopadhyay, A. Dutta, M. Faizal, EPL 112 (2015) 20006.

[24] M. Faizal, Phys. Lett. B 757 (2016) 244.

[25] S. Masood, M. Faizal, Z. Zaz, A.F. Ali, J. Raza, M.B. Shah, Phys. Lett. B 763 (2016) 218.

[26] C.-W. Chen, W.-Y. Wen, arXiv:1607.02690 [quant-ph].

[27] A. Kempf, G. Mangano, R.B. Mann, Phys. Rev. D 52 (1995) 1108.

[28] P. Pedram, Phys. Rev. D 85 (2012) 024016.

[29] E. Rudnicki, J. Phys. A: Math. Theor. 49 (2016) 375301.

[30] S. Pramanik, M. Moussa, M. Faizal, A.F. Ali, Ann. Phys. 362 (2015) 24.

[31] K. Abdelkhalek, W. Chemissany, L. Fiedler, G. Mangano, R. Schwonnek, Phys. Rev. D 94 (2016) 123505.

[32] E.H. Kennard, Z. Phys. 44 (1927) 326.

[33] H.P. Robertson, Phys. Rev. 34 (1929) 163.

[34] I. Białynicki-Birula, Z. Białynicki-Birula, Phys. Rev. Lett. 108 (2012) 140401.

[35] I. Białynicki-Birula, Z. Białynicki-Birula, Phys. Rev. A 86 (2012) 022118.

[36] I. Białynicki-Birula, Z. Białynicki-Birula, Phys. Rev. A 88 (2013) 052103.

[37] D. Deutsch, Phys. Rev. Lett. 50 (1983) 631.

[38] H. Maassen, J.B.M. Uffink, Phys. Rev. Lett. 60 (1988) 1103.

[39] K. Kraus, Phys. Rev. D 35 (1987) 3070.

[40] M. Krishna, K.R. Parthasarathy, Sankhyā Ser. A 64 (2002) 842.

[41] S. Wehner, A. Winter, New J. Phys. 12 (2010) 025009.

[42] I. Białynicki-Birula, Ł. Rudnicki, Entropic uncertainty relations in quantum physics, K.D. Sen, ed., Statistical Complexity, Springer, Berlin, 2011, p. 1.

[43] P.J. Coles, M. Berta, M. Tomamichel, S. Wehner, Rev. Mod. Phys. 89 (2017) 015002.

[44] S. Zozor, G.M. Bosyk, M. Portesi, J. Phys. A: Math. Theor. 47 (2014) 495302.

[45] Y. Huang, Phys. Rev. A 86 (2012) 024101.

[46] L. Maccone, A.K. Pati, Phys. Rev. Lett. 113 (2014) 260401.

[47] Z. Puchała, Ł. Rudnicki, K. Życzkowski, J. Phys. A: Math. Theor. 46 (2013) 272002.

[48] S. Friedland, V. Gheorghiu, G. Gour, Phys. Rev. Lett. 111 (2013) 230401.

[49] Ł. Rudnicki, Z. Puchała, K. Życzkowski, Phys. Rev. A 89 (2014) 052115.

[50] E. Rudnicki, Phys. Rev. A 91 (2015) 032123.

[51] A.E. Rastegin, K. Życzkowski, J. Phys. A: Math. Theor. 49 (2016) 355301.

[52] J. Kaniewski, M. Tomamichel, S. Wehner, Phys. Rev. A 90 (2014) 012332.

[53] I.I. Hirschman, Am. J. Math. 79 (1957) 152.

[54] W. Beckner, Ann. Math. 102 (1975) 159.

[55] I. Białynicki-Birula, J. Mycielski, Commun. Math. Phys. 44 (1975) 129.

[56] Y. Huang, Phys. Rev. A 83 (2011) 052124.

[57] M. Berta, M. Christandl, R. Colbeck, J.M. Renes, R. Renner, Nature Phys. 6 (2010) 659.

[58] F. Furrer, M. Berta, M. Tomamichel, V.B. Scholz, M. Christandl, J. Math. Phys. 55 (2014) 122205.

[59] P. Pedram, Adv. High Energy Phys. 2016 (2016) 5101389.

[60] S. Kawamoto, L.-Y. Hsu, W.-Y. Wen, arXiv:1605.04783 [quant-ph].

[61] R.R. Parwani, Ann. Phys. 315 (2005) 419.

[62] D. Mondal, S. Bagchi, A.K. Pati, arXiv:1607.06712 [quant-ph].

[63] R. de la Madrid, A. Bohm, M. Gadella, Fortschr. Phys. 50 (2002) 185.

[64] J. Distler, S. Paban, Phys. Rev. A 87 (2013) 062112.

[65] A.E. Rastegin, Ann. Phys. (Berlin) 528 (2016) 835.

[66] H.M. Wiseman, G.J. Milburn, Quantum Measurement and Control, Cambridge University Press, Cambridge, 2010.

[67] K. Baek, W. Son, Sci. Rep. 6 (2016) 30228.

[68] L.N. Chang, D. Minic, N. Okamura, T. Takeuchi, Phys. Rev. D 65 (2002) 125027.

[69] G.H. Hardy, J.E. Littlewood, G. Polya, Inequalities, Cambridge University Press, London, 1934.

[70] I. Białynicki-Birula, Phys. Lett. A 103 (1984) 253.

[71] A. Rényi, Proceedings of the 4th Berkeley Symposium on Mathematical Statistics and Probability, University of California Press, Berkeley, CA, 1961, p. 547.

[72] C. Tsallis, J. Stat. Phys. 52 (1988) 479.

[73] I. Bengtsson, K. Życzkowski, Geometry of Quantum States: An Introduction to Quantum Entanglement, Cambridge University Press, Cambridge, 2006.

[74] A.E. Rastegin, Found. Phys. 45 (2015) 923.

[75] I. Białynicki-Birula, Phys. Rev. A 74 (2006) 052101. 
[76] A.E. Rastegin, J. Phys. A: Math. Theor. 44 (2011) 095303. 